\title{
EXPERIMENTELLE THERAPIEENTWICKLUNG FÜR DIE AKUTE LYMPHATISCHE LEUKÄMIE
}

\author{
Susanne Fischer
}

\section{Zusammenfassung}

Bei Leukämie handelt es sich um eine Entartung von Vorläuferzellen der weißen Blutzellen (Leukozyten). Die hier betrachtete Form dieser Erkrankung ist die akute lymphatische Leukämie (ALL), bei der vor allem Rückfallpatienten eine schlechte Diagnose haben. Demnach wird nach neuen Therapien gesucht, die neue Angriffspunkte für die Behandlung der ALL bieten. Die getestete Substanz 5-Azacytidin hat zwei therapeutische Wirkmechanismen. Zum einen das Hervorrufen des selbstinduzierte Zelltod (Apoptose) und zum anderen einen epigenetischen Effekt. Der epigenetische Effekt bewirkt eine Veränderung der Genregulation (Veränderung der Genaktivität) ohne die DNASequenz zu beeinflussen. Dies kann mithilfe eines Screenings (Micoarraytechnologie) in behandelten und unbehandelten Proben verglichen werden. Da das Screening sehr global ist, erfolgt für eine Auswahl therapeutisch relevanter Gene eine Verifizierung mittels einer molekularbiologischen Methode (qRT-PCR). Dabei wurden Gene mit erhöhter Aktivität bestätigt, MAEL; TMEM56; IL1B; BCL6; MS4A1 und FLT3. Für jedes dieser Gene konnte ein positiver Nutzen für die Therapie einer ALL diskutiert werden. Für FLT3 erfolgte sogar eine positive Kombination mit einer anderen therapeutischen Substanz. Der therapeutische Einsatz des 5-Azacytidins kann zukünftig eine wichtige Therapieoption für Patienten mit ALL darstellen.

\section{Abstract}

Leukemia is a clonal disorder of the bone marrow resulting in degenerated precursors of white blood cells (leukocytes). This study reports about acute lymphoblastic leukemia (ALL). Especially, relapsed ALL patients have a bad diagnosis. In order to improve the chances for these patients, new targeted therapies need to be developed. The analyzed substance, 5-azacytidine, hast two main therapeutic effects. On the one hand it causes the self-induced cell death (apoptosis) and on the other hand there is an epigenetic effect. The epigenetic effect causes a change in gene regulation (change in gene activity) without affecting the DNA sequence. With the help of a screening (microarray technology), comparing treated and untreated samples changes in gene activity were detected. For the verification of therapeutic relevant genes (MAEL, TMEM56, IL1B, BCL6, MS4A1 and FLT3) a molecular biological technique (qRT-PCR) was used. Herein, for each of these genes a new therapeutic option is discussed. In addition, a combination of 5-azacytidine and another therapeutic substance (targeting FLT3) was tested. In the future, the therapeutic benefits of 5-azacytidine may be an important treatment option for patients suffering from ALL.

\section{»I. EINLEITUNG}

In Deutschland erkranken jährlich ca. 10.000 Menschen an Leukämie. Damit machen Leukämien einen Anteil von $2,1 \%$ aller Krebserkrankungen in Deutschland aus (medac). Bei der hier betrachteten akuten Form einer Leukämie handelt es sich um eine hochgradig maligne Erkrankung. Bei dieser Erkrankung entarten Vorläuferzellen der Leukozyten (der weißen Blutzellen) zu sogenannten unausgereiften Blasten und infiltrieren die blutbildenden Organe und die Blutbahnen. Diese akute Erkrankung führt ohne intensive Chemotherapie binnen weniger Wochen zum Tod des Patienten (cancerquest). Im Folgenden wird hier die ALL betrachtet. Bei dieser Erkrankung sind Vorläuferzellen der B- oder T-Lymphozyten entartet und es gibt eine zweigipflige Altersverteilung. Dabei ist sie zum einen die häufigste Krebserkrankung bei Kindern und gleichzeitig findet sich ein gehäuftes Auftreten der Erkrankung im höheren Lebensalter (Ribera 2011). Im Kindesalter liegen die Heilungsraten der ALL bei etwa $90 \%$. Problematisch gestaltet sich dabei, dass es bei mehr als $20 \%$ der jungen Patienten zu einem Rückfall kommt. Die Heilungsraten nach einem Rückfall sind wesentlich schlechter als bei einer Erstdiagnose (Bhatla et al. 2012). Die komplette Remission der ALL im Erwachsenenalter kann derzeit bei $85-90 \%$ der Patienten erreicht werden. Die Langzeitüberlebensrate liegt aber nur bei etwa 40-50\%. Auch hier sind Verschlechterungen der Heilungsraten bei einem Rückfall zu verzeichnen (Gökbuget et al. 2012). Als mögliche Therapieoption wurde für diese Studie eine Substanz eingesetzt, deren positive therapeutische Wirkung für akute myeloische Leukämie bereits klinisch eingesetzt wird. Es handelt sich dabei um die Substanz 5-Azacytidin, welche sowohl zytotoxisch auf Leukämiezellen wirkt sowie auch einen epigenetischen Wirkmechanismus, die DNA-Methylierung, aufweist. Unter Epigenetik versteht man einen Bereich der Molekularbiologie, der sich mit Modifikationen von Genen in einer Zelle beschäftigt. Diese Modifikationen erfolgen ohne Beeinflussung der DNA-Sequenz und sind reversibel. In diesem Fall entfernt 5-Azacytidin Methylierungen, die an einer spezifischen Base der DNA (dem Cytosin) angebracht sein können. Dazu interferiert es als RNA-Baustein bei der Transkription oder wird als umgewandelte Desoxy-Form direkt in die DNA eingebaut. Dort wird es für DNA-Methyltransferasen (DNMTs) zum Substrat. Im Vergleich zu normalen Cytosin-Nukleotiden bindet 5-Azacytidin die DNMTs kovalent, sodass diese nicht abdissoziieren 
können und somit inaktiviert sind. Bei den folgenden DNA-Replikationen wird dann an dieser Stelle keine Methylgruppe mehr übertragen (Leone et al. 2002). Dieses Methylierungsmuster ist bei jedem Menschen unterschiedlich und vor allem bei malignen Erkrankungen stark verändert. Wichtige Grundmechanismen der Zellen sind dadurch verändert und ändern somit auch die Funktionalität der Zellen (Piekarz und Bartes 2009). Veränderungen des Methylierungsmusters durch epigenetische Substanzen wie 5-Azacytidin führen somit zu neuerlich veränderten Expressionsmustern in den malignen Zellen. Dementsprechend können in dem so veränderten Expressionsmuster Gene untersucht werden, deren Auftreten einen neuen und geeigneten therapeutischen Ansatz bei der Behandlung der ALL darstellen. Infolgedessen wurden für diese Arbeit Affymetrix-Daten erhoben, die einen Vergleich der Genexpression von unbehandelten und behandelten (5-Azacytidin) ALL Zelllinien (Nalm-6 und BE13) ermöglichen. Auf der Grundlage dieser Daten konnten nun differentiell exprimierte Gene analysiert werden. Da die Auswertung der GeneChips mit einer Normalisierung über den gesamten Chip einhergeht, können fehlerhafte Expressionen auftreten. Darum ist es unbedingt notwendig diese Ergebnisse nur als Grundlage für weitere Untersuchungen anzusehen.

Eine Verifizierung der Ergebnisse erfolgte daher mittels qRT-PCR.

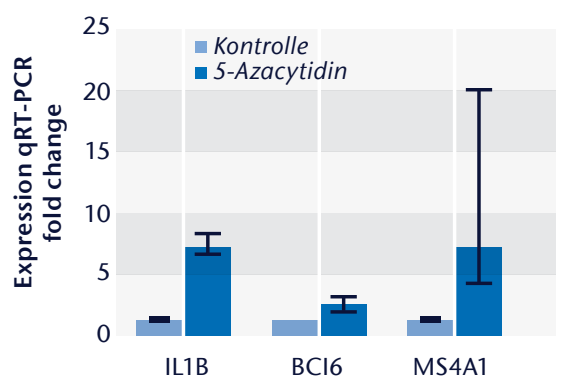

Abb. 1a) Ergebnis der qRT-PCR für IL1B, BCL6 und MS4A1 in Nalm-6 Zellen. Für einen Versuch (pro Gen) wurden jeweils drei Kontrollen und drei Proben (5-Azacytidin 1,3 $\mu \mathrm{M}$ ) dargestellt. Zudem zeigt die Grafik die Veränderung des fold changes (auf der Ordinate als Expression qRT-PCR angegeben) der Proben gegenüber den Kontrollen. Die Kontrollen sind dabei auf 1 normiert. Die Fehlerbalken geben die minimale und maximale Abweichung vom Mittelwert an.

\section{» II. METHODEN}

Die Verifizierung der ermittelten Kandidatengene erfolgte mit etablierten molekularbiologischen Methoden. Zunächst erfolgte dabei eine Kultivierung der etablierten Zelllinien Nalm- 6 und BE13 bei $4 \times 10^{5}$ Zellen $/ \mathrm{ml}, 37^{\circ} \mathrm{C}$ und $5 \% \mathrm{CO}_{2}$. Diese wurden dann über 72 Stunden mit 5-Azacytidin behandelt (Nalm-6: 1,3 $\mu \mathrm{M}$ und BE13 $1 \mu \mathrm{M}$ ). Als Referenzkontrolle galten Zellen, die mit dem Lösungsmittel des 5-Azacytidins (Dimethylsulfoxid) behandelt wurden. Aus dem gewonnenen Zellmaterial konnte dann die RNA isoliert werden und im Anschluss in cDNA (complementary DNA) umgeschrieben werden. Diese dient als Template für die qRT-PCR (quantitativ real-time polymerase chain reaction). Die Auswertung der Ergebnisse erfolgte dann nach der $2^{-\triangle \Delta C T}$ von Schmittgen und Livak, 2008. Bei den Kombinationsversuchen von 5-Azacytidin und Sorafenib wurden die Zellen zunächst mit 5-Azcytidin und anschließend mit Sorafenib behandelt. Die Auswertung erfolgte mittels der Proliferationsreagenz WST-1.

Zellen mit einer gesunden Stoffwechselaktivität können das rot gefärbte Tetrazoliumsalz WST-1 in Formazan umzusetzen (Peskin und Winterbourn 2000). Dieser Farbumschlag kann dann bei 450 $\mathrm{nm}$ photometrisch bestimmt werden.

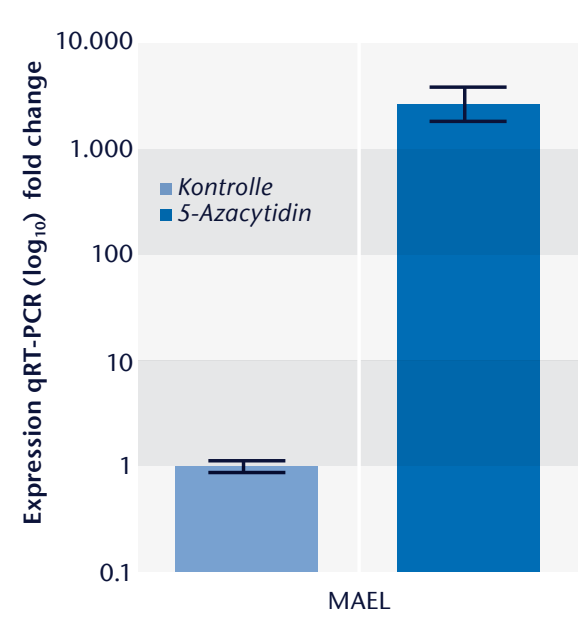

Abb. 1b) Ergebnis der qRT-PCR für MAEL in BE13 Zellen Für einen Versuch wurden jeweils drei Kontrollen und drei Proben (5-Azacytidin $1 \mu \mathrm{M}$ ) dargestellt. Zudem zeigt die Grafik die Veränderung des fold changes (auf der Ordinate als logarithmische Expression GRT-PCR angegeben) der Proben gegenüber den Kontrollen. Die Kontrollen sind dabei auf 1 normiert. Die Fehlerbalken geben die minimale und maximale Abweichung vom Mittelwert an.

\section{» III. ERGEBNISSE}

Die mittels Affymetrix-Daten ermittelten Dysregulationen wurden durch qRTPCR verifiziert. Dazu musste zunächst für jedes Kandidatengen ein Primerpaar desingt und getestet werden. Danach erfolgte die qRT-PCR. Anhand der fold change Analyse nach Schmittgen und Livak (2008) konnten relative Expressionen der behandelten Proben gegenüber den unbehandelten Kontrollproben ermittelt werden. Dabei war auffällig, dass bei der B-ALL (Zelllinie Nalm-6) und T-ALL (Zelllinie BE13) unterschiedliche Gene dysreguliert waren. Es gab jedoch auch eine Schnittmenge von 109 gemeinsam hochregulierten Genen. Für drei der ausgewählten Kandidatengene (BCL6, IL1B und MS4A1) konnten die Dysregulationen nur in B-ALL also Nalm-6 Zellen nachgewiesen werden. B-ALL haben einen Anteil von $80 \%$ aller ALL Erkrankungen, deshalb ist eine separate Betrachtung dieser Erkrankung durchaus sinnvoll. Die anderen Kandidatengene MAEL und TMEM56 sind in beiden Zelllinien nach der Behandlung mit 5-Azacytidin verstärkt exprimiert. In Abb. 1a sind die Ergebnisse der qRT-PCR für die in Nalm-6 hochregulierten Kandidatengene dargestellt.

Die ermittelten Ergebnisse ließen sich reproduzieren und konnten mindestens als signifikant eingestuft werden (nicht dargestellt). Eine besonders starke Dysregulation weist MAEL auf. Sowohl in Nalm-6 als auch in BE13 (Abb. 1b) Zellen kann eine enorme Expressionserhöhung verzeichnet werden. Da es sich bei ALL um eine sehr heterogene Erkrankung handelt, kommen für eine Therapie immer Substanzkombinationen zum Einsatz. Das für die Substanz Sorafenib bekannte Targetgen FLT3 war in den mittels Affymetrix erhobenen Daten ebenfalls hochreguliert. Auch hier konnte die erhöhte Expression per qRT-PCR verifiziert werden (Abb. 2).

Sorafenib ist ein Multi-Kinase-Inhibitor und wird klinisch bei der Behandlung von schwerem Nieren- oder Leberkrebs eingesetzt. Der Einsatz von Sorafenib führt dazu, dass die Proliferation der Tumorzellen herabgesetzt wird (Pharmazeutische Zeitung). Bei der Kombination von Sorafenib und 5-Azacytidin konnte sowohl in BE13 als auch in Nalm-6 Zellen 


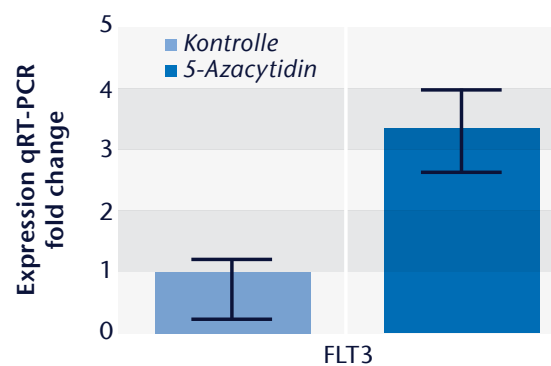

Abb. 2) Ergebnis der qRT-PCR für FLT3 in Nalm-6 Zellen. Ein Versuch mit jeweils drei Kontrollen und drei Proben (5-Azacytidin 1,3 $\mu \mathrm{M}$ ) ist dargestellt. Zudem zeigt die Grafik die Veränderung des fold changes (auf Ordinate als Expression qRT-PCR angegeben) der Proben gegenüber den Kontrollen. Die Kontrollen sind dabei auf 1 normiert. Die Fehlerbalken geben die minimale und maximale Abweichung vom Mittelwert an.

ein positiver Kombinationseffekt ermittelt werden. Für Nalm- 6 ist das Ergebnis der Kombination in Abb. 3 dargestellt.

Wie in Abb. 3 zu erkennen, liegt die Viabilität der Zellen (Nalm-6) nach einer Behandlung mit Sorafenib ( $4 \mu \mathrm{M})$ bei $90 \%$. Die Konzentrationen von 5-Azacytidin wurden in diesem Versuch variiert. Die rote Kurve gibt den Verlauf der Einzelkonzentrationen von 5-Azacytidin wieder. Die blaue Kurve verdeutlicht den Verlauf der Effekte verschiedener 5-Azacytidinkonzentrationen mit $4 \mu \mathrm{M}$ Sorafenib. So kann beispielsweise bei einer Konzentration von $2 \mu \mathrm{M}$ 5-Azacytidin eine Viabilität von $86 \%$ gemessen werden. Die Viabilität von $4 \mu \mathrm{M}$ Sorafenib liegt, wie bereits beschrieben, bei $90 \%$. Durch die Kombination beider Substanzen proliferieren jedoch nur noch $62 \%$ der Zellen.

Die Viabilität bezeichnet hier die anteilig lebenden Zellen in einer Zellpopulation. Die Kombination von 5-Azacytidin und Sorafenib zeigt sehr gute Kombinationseffekte (Abb. 3). Die erhöhte Expression eines Targetrezeptors (von Sorafenib) mittels 5-Azacytidin ist somit ein guter Ansatz für eine Therapie mit Sorafenib.

\section{»IV DISKUSSION}

Die hier durchgeführten Untersuchungen erfolgten in Kooperation mit dem Forschungsbereich der hämatologischen und onkologischen Abteilung der Charité Berlin-Steglitz. Die Ergebnisse wurden im Rahmen einer Masterarbeit des Studiengangs Biosystemtechnik/Bioinformatik der TH Wildau [FH] erhoben. Wie bereits für das Targetgen FLT3 gezeigt, werden immer Substanzkombinationen für die Therapie einer ALL eingesetzt. Für die 5 weiteren Kandidatengene gibt es dabei spezifische Kombinationsmöglichkeiten.

Die erhöhte Expression des BCL6 scheint auf den ersten Blick problematisch, denn BCL6 hemmt das natürliche Reparatursystem der DNA und ermöglicht somit das Überleben von malignen Zellen (Ranuncolo et al, 2007). Jedoch ist es auch eine Targetstruktur für einen anti-BCL6inhibitor. Weiterführend wäre somit zu untersuchen, inwiefern eine Vorbehandlung der Zellen mit 5-Azacytidin, also eine Überexpression von BCL6, die Wirksamkeit des anti-BCL6 Inhibitors verstärkt. IL1B ist ein Entzündungsmediator und Botenstoff, welcher Einfluss auf verschiedenste Bereiche der Zellregulation hat. Dazu zählen Zellproliferation, Zelldifferenzierung und auch die Apoptose (cancer). Die erhöhte Expression des IL1B nach 5-Azacytidinbehandlung kann zu einer Aktivierung des körpereigenen Immunsystems führen, welches die Bekämpfung der malignen Zellen durch körpereigene Abwehrmechanismen anregt. Für das Oberflächenantigen MS4A1 (auch als CD20 bekannt) gibt es bereits eine zugelassene Therapie. MS4A1 ist B-Zell spezifisch und kann mithilfe des chimären Antikörpers Rituximab angegriffen werden. Eine Erhöhung der Oberflächenantigene durch die Behandlung mit 5-Azacytidin erhöht somit die Anzahl an Targetgenen für Rituximab. Bei TMEM56 handelt es sich ebenfalls um ein Oberflächenantigen, das einen potentiellen Einsatz als Targetstruktur ermöglicht. Es gibt jedoch kaum Informationen über die Funktion von TMEM56. Hierfür müsste zunächst untersucht werden, ob es ausschließlich auf malignen Zellen eine starke Expression aufweist. Dies konnte für MAEL bereits gezeigt werden. Xiao et al. (2010) berichteten, das MAEL in normalem Gewebe nicht exprimiert wird. In maligen Zellen dagegen ist die Expression erhöht und nach einer demethylierenden Behandlung gibt es eine enorme Expressionserhöhung. Diese konnte auch in dieser Studie nach Behandlung mit 5-Azacytidin gezeigt werden (vergl. Abb. 1b). Somit ist MAEL ein geeignetes Tumorantigen, welches $z$. B. als Tumormarker Verwendung finden kann. Einen Überblick über die dargestellten Therapieoptionen gibt Tabelle 1. Sie zeigt zunächst einen Überblick aller Kandidatengene und ihrer potentiellen Funktionen für die Therapie einer ALL. Weiter gibt sie eine Übersicht, in welchen Zelllinien das jeweilige Gen durch 5-Azacytidinbehandlung hochreguliert werden konnte. Auch die Zuordnung in verschiedene Gengruppen und der mögliche therapeutische Einsatz, sind dargestellt.

\section{» V ZUSAMMENFASSUNG}

Durch die Behandlung von ALL Zelllinien mit 5-Azacytidin kann die Auftrittshäufigkeit einzelner Gene verändert werden (differentielle Genexpression). In dieser Arbeit wurden beispielhaft

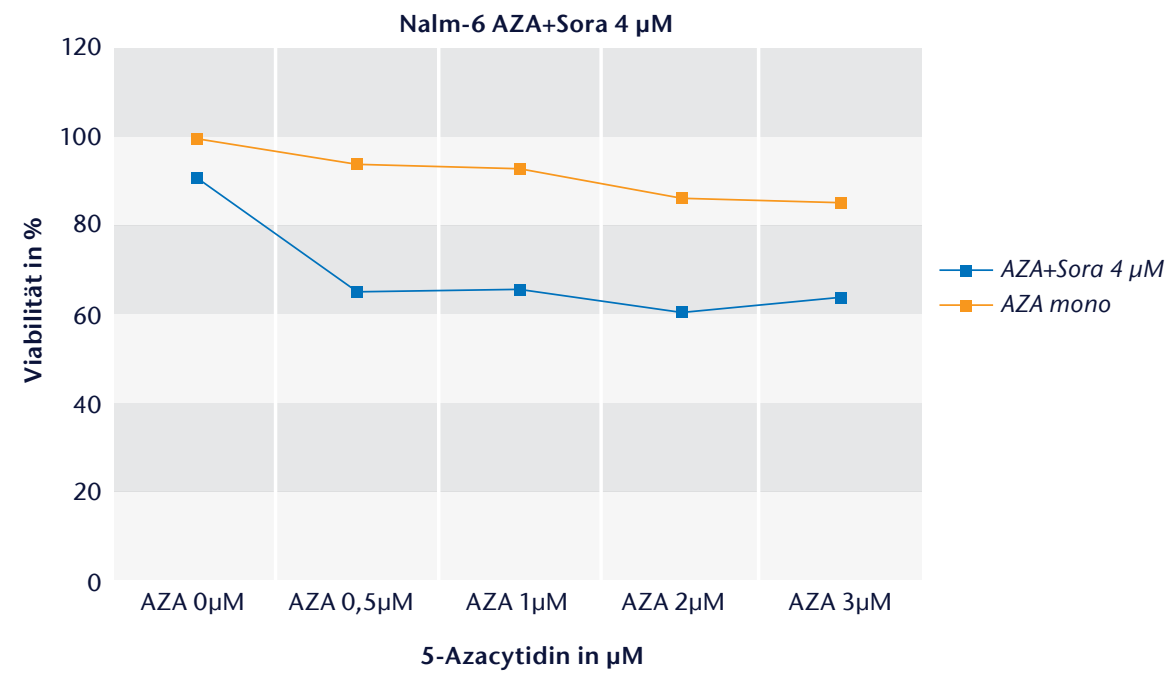

Abb. 3) Viabilitätsmessung von Nalm-6 nach Inkubation mit 5-Azacytidin und Sorafenib. Die Abszisse entspricht den 5-Azacytidinkonzentrationen in $\mu \mathrm{M}$, die Ordinate der Viabilität in Prozent. Die Abnahme der Viabilität kann durch Einsatz von 5-Azacytidin und Sorafenib mono, aber auch in Kombination gezeigt werden. 
Kandidatengene ausgewählt, für die zusätzlich eine mögliche Kombinationstherapie getestet oder diskutiert werden kann. Der positive Kombinationseffekt des 5-Azacytidins mit Sorafenib zeigt das Potential der Substanz, da Kombinationstherapien für die Behandlung von Leukämien grundlegend sind. Zudem wird durch 5-Azacytidin ein

\begin{tabular}{|c|c|c|c|}
\hline & Hochreguliert in & $\begin{array}{l}\text { Zuordnung zu } \\
\text { relevanten Gengruppen }\end{array}$ & $\begin{array}{l}\text { Möglicher } \\
\text { therapeutischer Einsatz }\end{array}$ \\
\hline BCL6 & Nalm-6 (B-ALL) & Onkogen & $\begin{array}{l}\text { Targetgen für den } \\
\text { anti-BCL6 Inhibitor }\end{array}$ \\
\hline IL1B & Nalm-6 (B-ALL) & Entzündungsmarker & $\begin{array}{l}\text { Aktivierung des körper- } \\
\text { eigenen Immunsystems }\end{array}$ \\
\hline MS4A1 & Nalm-6 (B-ALL) & Oberflächenantigen & $\begin{array}{l}\text { Antikörpertherapie } \\
\text { mittels Rituximab }\end{array}$ \\
\hline TMEM56 & $\begin{array}{l}\text { Nalm-6 (B-ALL) } \\
\text { BE13 (T-ALL) }\end{array}$ & $\begin{array}{l}\text { Mögliches Tumoranti- } \\
\text { gen }\end{array}$ & $\begin{array}{l}\text { Impfstrategien, } \\
\text { Antikörpertherapie }\end{array}$ \\
\hline$\overline{\text { MAEL }}$ & $\begin{array}{l}\text { Nalm-6 (B-ALL) } \\
\text { BE13 (T-ALL) }\end{array}$ & Tumorantigen & $\begin{array}{l}\text { Impfstrategien, } \\
\text { Tumormarker }\end{array}$ \\
\hline
\end{tabular}

Tabelle 1) Übersicht über die ausgewählten Kandidatengene und ihre möglichen Einsatzmöglichkeiten bei der Therapie von ALL. Zudem ist dargestellt, in welcher Zelllinie eine Hochregulation des jeweiligen Gens nach 5-Azacytidinbehandlung zu detektieren ist. zelleigener Mechanismus aktiviert, der den selbstprogrammierten Zelltod der Zelle einleitet (Apoptose). Die Ergebnisse dieser Studie zeigen, dass der Einsatz von 5-Azacytidin für die Behandlung einer ALL durchaus positive Effekte erzielt und eine neue Therapieoption für Patienten mit ALL darstellt.
LITERATUR

Bhatla T., Wang I., Morrison D. J., Raetz E. A., Burke MI, Brown P., Carroll W. L. (2012): „Epigenetic reprogramming reverses the relapse-specific gene expression signature and restores chemosensitivity in childhood B-lymphoblastic leukemia." In: Blood. 2012 May 31; 119(22): 5201-10. Epub 2012 Apr 11. (New York University Cancer Institute, New York University Langone Medical Center, New York, NY 10016, USA) cancer:

http://www.cancer.gov/dictionary?cdrid=624952 (National Cancer Institute), Zugriff am 20.08.2012.

cancerquest: http://www.cancerquest.org/types-ofleukemia.html (Winship Cancer Institute), Zugriff am 11.05.2012.

Gökbuget N., Stanze D., Beck J., Diedrich H., Horst H. A., Hüttmann A., Kobbe G., Kreuzer K. A., Leimer L., Reichle A., Schaich M., Schwartz S., Serve H., Starck M., Stelljes M., Stuhlmann R., Viardot A., Wendelin K., Freund M., Hoelzer D. (2012): „Outcome of relapsed adult lymphoblastic leukemia depends on response to salvage chemotherapy, prognostic factors and realization of stem cell transplantation." In: Blood. 2012 Apr 4. PubMed PMID: 22493293. (Department of Medicine II, Goethe University Hospital, Frankfurt, Germany).

Leone, G., Teofili, L., Voso, M. T., Lübert, M. (2002): „DNA methylation and demethylating drugs in myelodysplastic syndromes and secondary leukemias." In: Haematologica 2002; 87: 1324-1341 (Institute of Hematology, Catholic University, Rome, Italy). medac: http://www.medac.de/ patient/fachbereiche/haematologie/index_pr.htm, Zugriff am 12.09.2012.

Peskin, A. V., Winterbourn, C. C. (2000): „A microtiter plate assay for superoxide dismutase using a water-soluble tetrazolium salt (WST-1)." In: Clinica Chimica Acta 293: 157-166 (Free Radical Research Group, Department of Pathology, Christchurch School of Medicine, P. O. Box 4345, Christchurch, New Zealand)

Piekarz, R. L., Bates, S. E,: (2009) „EpigeneticModifiers: Basic Understanding and Clinical Development." In: Clin Cancer Res 2009; 15 (12) (Medical Oncology Branch, Center for Cancer Research, National Cancer Institute, Bethesda, Maryland 20892, USA.).

Pharmazeutische Zeitung:

http://www.pharmazeutische-zeitung.de/index. php?id=2511, Zugriff am 15.09.2012.

Ranuncolo, S. M., Polo, J. M., Dierov, J., Singer, M., Kuo, T., Greally, I., Green, R., Carroll, M., Melnick, A. (2007): „BCl-6 mediates the germinal center $\mathrm{B}$ cell phenotype and lymphomagenesis through transcriptional repression of the DNA-damage sensor ATR." In: Nature Immunology 8, 705 - 714 (2007) (Department of Developmental and Molecular Biology, Albert Einstein College of Medicine, Bronx, New York 10461, USA)

Ribera, J.-M. (2011) „Acute lymphoblastic leukemia in adults" In: Pediatric Reports 2011; volume 3:(s2)e1 (Clinical Hematology Department, Institut Catala d'Oncologia, Hospital Universitari Germans Trias i Pujol, Badalona, Spain).

Schmittgen, T. D., Livak, K. J. (2008): „Analyzing real-time PCR data by the comparative C(T) method.“ In: Nat Protoc. 2008; 3 (6): 1101-8. (Division of Pharmaceutics, College of Pharmacy, Ohio State University, Parks Hall, 500 West 12th Avenue, Columbus, Ohio, OH 43210 USA).

Xiao L., Wang Y., Zhou Y., Sun Y., Sun W., Wang L., Zhou C., Zhou J., Zhang J. (2010): „Identification of a novel human cancer/testis gene MAEL that is regulated by DNA methylation." In: Mol Biol Rep. 2010 Jun; 37 (5): 2355-60. Epub 2009 Aug 20. (Key Laboratory of Protein Chemistry and Developmental Biology of Education Ministry of China, College of Life Science, Hunan Normal University, Changsha, Hunan 410081, China).

AUTOR

M.sc. Susanne Fische

Biosystemtechnik/Bioinformatik

TH Wildau [FH]

susanne.fischer86@web.de 